Journal of Mathematics and Informatics

Vol. 17, 2019, 21-29

ISSN: 2349-0632 (P), 2349-0640 (online)

Published 3 June 2019

www.researchmathsci.org

DOI: http://dx.doi.org/10.22457/jmi.130aav17a3

Journal of

Mathematics and

Informatics

\title{
Application of Clusters in a Transportation Network
}

\author{
D.G.N.D. Jayarathna ${ }^{1}$ and C.J.Jayawardene ${ }^{2}$
}

${ }^{1}$ Faculty of Management, Humanities and Social Sciences, CINEC Campus, Sri Lanka

${ }^{2}$ Department of Mathematics, Faculty of Science, University of Colombo, Sri Lanka

${ }^{1}$ Corresponding author. Email: c_jayawardene@yahoo.com

Received 26 January 2019; accepted 30 April 2019

\begin{abstract}
The government of Sri Lanka has established several economic centres in the provincesbased on the budget proposal which was released in the year 1998. The Dambulla economic centre was the first such centre which was established on the 1st of April 1999. Since then, a number of economic centres were established throughout the island. However, Dambulla main hub continues to be the central warehouse where the vegetables are stored in the island. This paper deals with vehicle scheduling related to transportation and it investigates a method whereby a solution can be arrived, using linear programming (LP). Marketing Department Logistics (MDL), Ltd. needs to distribute vegetables and fruits to different provinces. Its main hub is situated near the Dambulla vegetable and fruit market and minor hubs are situated in different provinces in Sri Lanka. The main objective of this research is building a cost minimized model which creates a suitable method for delivering vegetables and fruits from the Dambulla major hub through its minor hubs to outlets in the provinces. Hence, in order to optimize the cost of outbound distribution, a mathematical model has been developed by using Integer Linear Programmingand by using industrial based reliable sources to collect data. Software assistance was obtained using the LINGO 06 optimizer, Java, MS Access and MS Excel tools to solve this mathematical model. This study is based on the Dambulla economic centre. This is an initial step to bring a correct protocol to arrange a transport model in order to distribute the vegetables and fruits from this centre in a cost-effective manner. According to this study, all districts in Sri Lanka could be divided into four clusters. At the beginning of this research, we assumed that each district contains two warehouses and three vendors. This model paves the way to create a larger model for solving any type of transportation planning problem.
\end{abstract}

Keywords: Vehicle scheduling, Minimizing transportation cost, Hamiltonian cycle, LINGO

AMS Mathematics Subject Classification (2010): 80M50, 13P25, 65K10

\section{Introduction}

Distribution systems can be defined as the sequential flow of procedures, systems, and activities which are designed and linked to facilitate and monitor the movement of goods and services from the source to the consumer. Essentially, distribution is about making products and services available to the end user when and where they need them. Often, 


\section{D.G.N.D.Jayarathna and C.J.Jayawardene}

you might hear the term 'channel of distribution' or 'marketing channel' with reference to distribution systems.

These two terms relate to a group of organizations or individuals who have an impact on the flow of products and services from the source of production to the end consumer. Since Sri Lanka is an agricultural country, its staple food is rice and curry. Therefore, vegetable and fruit consumption areusually very high among all Sri Lankans. In spite of the obvious variation in their production in different areas in the county, the demand for vegetables and fruits has remained constant, and is proportionate to the population density of each area. Provision of these goods to island-wide consumers is, therefore, a necessity. As the demand for these goods in different areas varies, the amount and frequency of delivering fresh fruits and vegetables need to be adjusted according to area demands. Delivery of those goods from the producer to the customer with a minimal price discrepancy in a cost-effective manner and via a minimally interrupted supply channel is called a fruit and vegetable distribution system. To make that a productive, cost effective process, the requirements of a neighborhood of a specific area of the country at a given time and the distance to thatparticular neighborhood need to be assessed. There should be a well-organized coordination between the economic centers and the peripheral warehouses. In Sri Lanka, the current distribution system operates in a manner where products are bought from the farmer by a middleman and delivered to the market or economic centerby the middlemen. They are delivered to warehouses located in many areas of the country using trucks, and sold at a wholesale price, after which they are supplied to vendors and finally to the consumers at a retail price (see $[2,4,5,8])$. There are some problems that arise in the system due to a poor understanding of the requirements of fruits and vegetables in some areas, and the absence of a good transportation schedule, and this results in an excess or deficit in the supply. Further, due to incorrect transportation practices, a considerable postharvest loss occurs. To overcome those problems, there is a need for a proper distribution system in Sri Lanka's fruit and vegetable market.

\section{Objectives of the research}

The main objective of this research is building a cost minimized model which creates a suitable method for delivering vegetables and fruits from the Dambulla major hub through its minor hubs to the outlets in the provinces.

In this research, the final goal is to build an Internet Based System to solve this mathematical problem. The LINGO solver has been used in two ways for two major parts of this system.

1. Using the Hamiltonian Cycle Algorithm to find the cluster-basedcycles for delivering vegetables and fruits from the Dambulla major hub to each warehouse located in different districtsof Sri Lanka.

2. Using Excel, Access and Java software to build a computer-based system which delineates the manner in which all vegetables and fruits can be delivered to all vendors from warehouses in Sri Lanka.

\section{Methodology and research techniques}

Solving an optimization model is a comprehensive task that requires a great deal of time for calculation (see $[1,6])$. But these models have economic value especially when considering the supply chain optimization. As a result, many logistics solution providers 
Application of Clusters in a Transportation Network

have emerged to cater to the rising demand, and these custom-made solutions are highly paid for by companies. At the same time, software has also been developed to facilitate these mathematical models to yield accurate calculations.

By coding the objective function and all the constraints in a particular programming language, the solution can be obtained faster and without errors. LINGO is one popular software tool among industrial professionals in transportation, logistics and finance for reliable modelling calculations.LINGO software facilitates optimization modelling in linear, nonlinear, quadratically constrained, second order cone, stochastic and integer models in an easier and faster way. It contains an integrated package that provides a powerful programming language for expressing optimization modelling and a fully functional software for building and editing problems.

\section{Route analysis - Hamiltonian cycle}

The first step of the research is to find a route plan with optimal path. It is implemented by using lingo software and modified/custom crafted traveling salesman's problem. Through that, we generated the optimal Hamiltonian related to each main cluster. Part of the modified Hamiltonian cycle sample problem for cluster 2 is given below.

MODEL:

!Traveling Salesman Problem for the cities ofcluster 2, Colombo 01, Colombo 09, Colombo 11, Colombo 12, Colombo 13, Colombo 14, Colombo 15;

SETS: CITY / 1..7/: V; !V(I) = sequence no. of city;

LINK(LOCALCITY, LOCALCITY):

DISTANCE ! The distance matrix;

$\mathrm{C} ; \mathrm{C}(\mathrm{I}, \mathrm{J})=1$ if we use link I, $\mathrm{J}$;

\section{ENDSETS}

\section{Connecting to the database}

Database connectivity to Access database is performed via a JdbcOdbc driver as shown below.

String database $=$ "jdbc:odbc:DRIVER $=\{$ MicrosoftAccessDriver

(*.mdb) $\} ; \mathrm{DSQ}=\mathrm{C}: 11$ Databases $\backslash \mathrm{DB} . \mathrm{mdb} "$;

Connection conn = DriverManager.getConnection(database, "", "'"); Statement stmt = conn.createStatement();

String query="SELECT $\bullet$ FROM Users WHERE Username=m+a+"' And Password = $1 "+c+"$;

\section{Detailed model assumptions}

We identified the fact that the objective of MDL is to reduce the transportation costs in delivering goods from its major hub through its minor hubs to its outlets in the provinces. Here the mathematical model for MDL was formulated by considering the following assumptions:

1. All districts of Sri Lanka are divided into four groups: Cluster 1; Cluster 2; Cluster 3; Cluster 4. 


\section{D.G.N.D.Jayarathna and C.J.Jayawardene}

2. Each district contains two warehouses. Locations of the warehouses and relevant distances will be applied to the model accordingly.

3. Each district contains three vendors. One vendor represents ten supermarkets in a certain area.

4. The volume capacity of trucks is assumed to be the same.

\section{Analysis of the research}

This research is based on building a cost optimization model which derives a suitable method for delivering vegetables and fruits from the Dambulla major hub through its minor hubs to its outlets in the provinces.

The final goal of this research is to build an Internet Based System to solve this mathematical problem. However, in order to satisfy these major points, the LINGO solver was used in two locations.

Advanced analysis was carried out utilizing the following tasks.

\section{Task 1}

I. All districts of Sri Lanka were divided into four groups: Cluster 1, Cluster 2, Cluster 3, Cluster 4.

Table 1: Details of clusters

\begin{tabular}{|c|c|c|c|}
\hline Index & Region & The districts contained & \\
\hline 1 & Cluster 1 & $\begin{array}{l}\text { Anuradhapura, Vavuniya, } \\
\text { Mannar, Jaffna, Kilinoch- } \\
\text { chi, Mullaitivu, Trincoma- } \\
\text { lee ... }\end{array}$ & $\begin{array}{l}\text { Kilinochchi Mullaittivu } \\
\text { Mannar Vavuniya }\end{array}$ \\
\hline 2 & Cluster 2 & $\begin{array}{l}\text { Puttalam, Kurunegala, } \\
\text { Kegalle, Colombo, Gam- } \\
\text { paha, Kalutara, Rathnapu- } \\
\text { ra, Galle, Matara... }\end{array}$ & $\begin{array}{l}\text { Trincomalee } \\
\text { Anuradhapura } \\
\text { Puttalam Plonnaruwa } \\
\text { Batticaloa }\end{array}$ \\
\hline 3 & Cluster 3 & $\begin{array}{l}\text { Matale, Kandy, Badulla, } \\
\text { Monaragala, Hambanthota, } \\
\text { Nuwara Eliya... }\end{array}$ & $\begin{array}{l}\text { Kampaha Kagalle } \\
\text { Colombo } \\
\text { Bawardy }\end{array}$ \\
\hline 4 & Cluster 4 & $\begin{array}{l}\text { Polonnaruwa, Batticaloa, } \\
\text { Ampara,.. }\end{array}$ & Kalutara Ratnapura Monarage \\
\hline
\end{tabular}

Task 2

Each district contains two warehouses and three vendors. A vendor represents ten supermarkets in a certain area. Locations of the warehouses and relevant distances will be applied to the model accordingly.

\section{Task 3}

Under this task, the Hamiltonian cycle was used to find the major paths related to each cluster which are used to deliver vegetables and fruits from the Dambulla major hub to each warehouse in every district in Sri Lanka. 
Application of Clusters in a Transportation Network

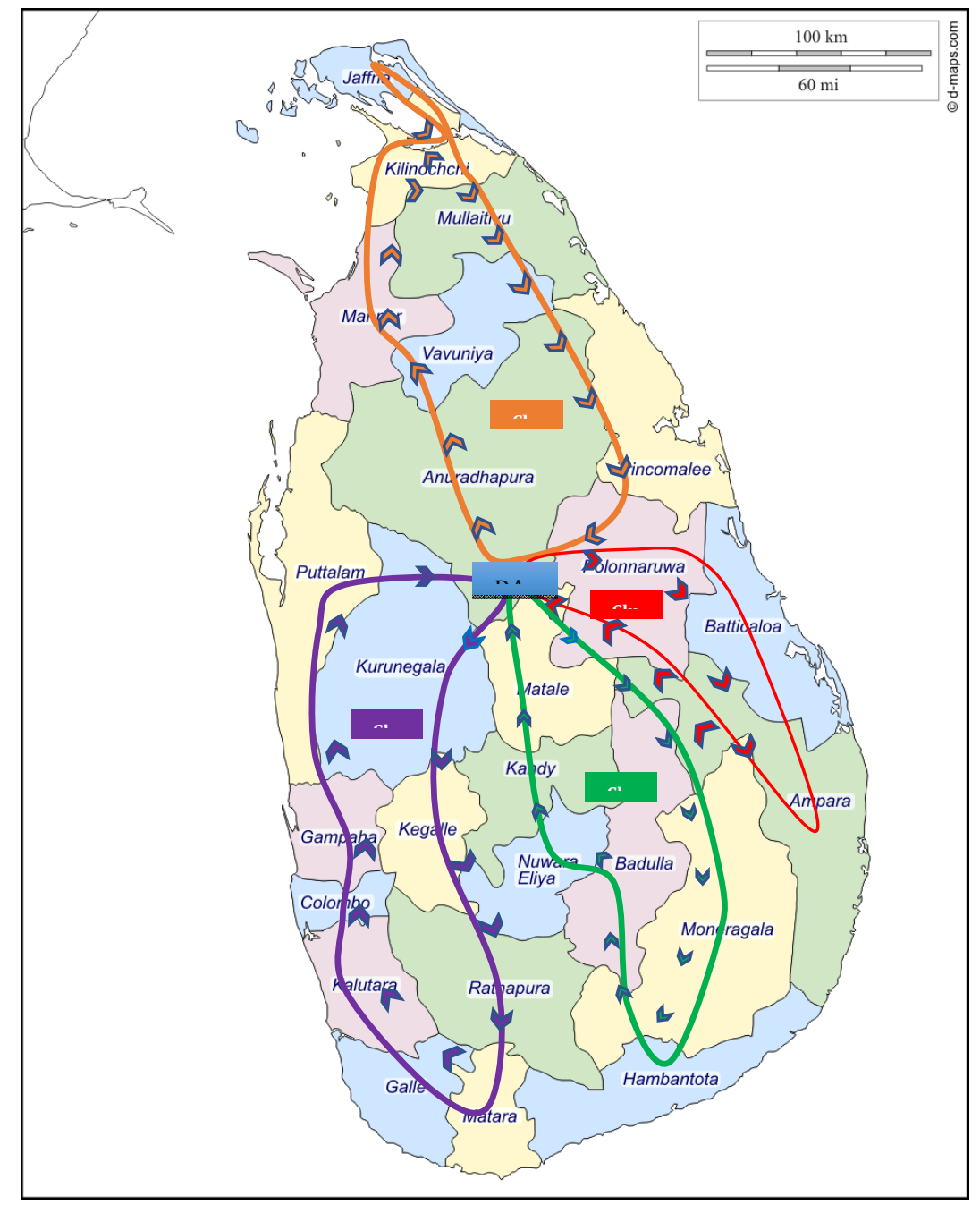

Figure 1: Hamiltonian path of each cluster

\section{Task 4}

During this step, Excel, Access and Java software were used to build a computer-based system which delineates the manner in which all vegetables and fruits are delivered to each vendor from all warehouses in Sri Lanka.

\section{Advanced analysis}

\section{Route analysis using Hamiltonian cycles}

The first step of the research is to find a route plan, where the optimal path is calculated using the LINGO software. LINGO is a software which facilitates the solution of linear and non-linear optimization problems. Through LINGO, the Hamiltonian cycle model is adjustedaccordingly to suit this problem. The LINGO output will yield the Hamiltonian cycle related to the optimal path to each cluster. 
D.G.N.D.Jayarathna and C.J.Jayawardene

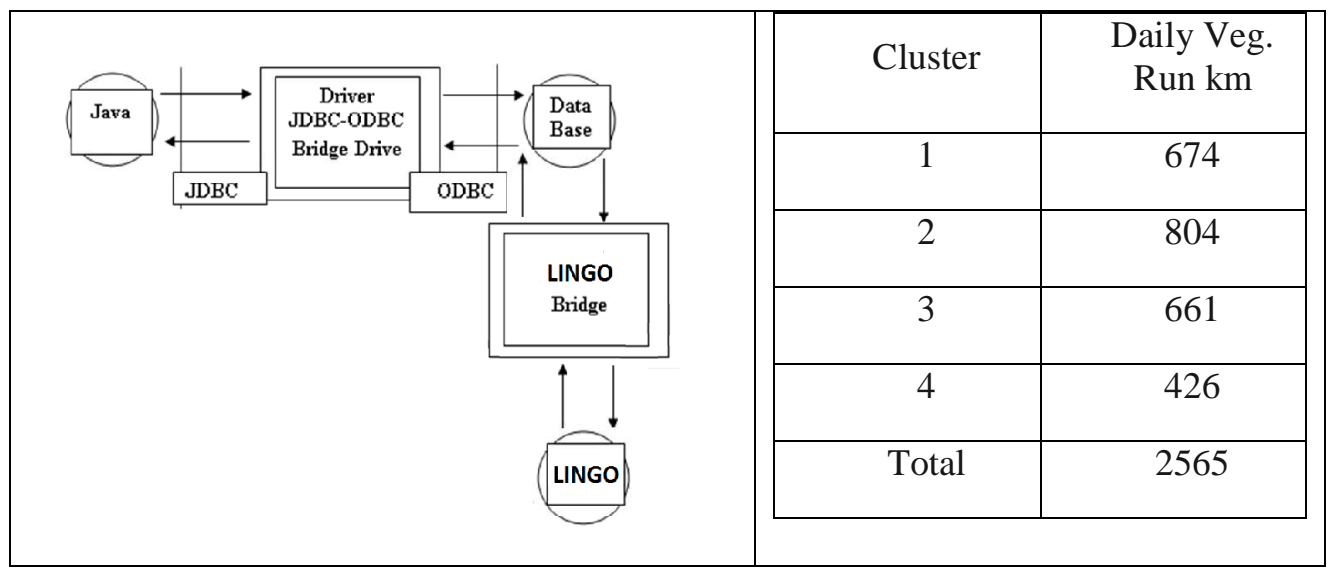

Table 2: Database connection and daily vegetable run in $\mathrm{km}$

Application of warehouse/vendor for food distribution within local provinces

In order to illustrate how the Warehouse/Vendor algorithm is applied, let us consider a small example in an Anuradhapura town which has two warehouses supplying three vendors with their tractors. Each warehouse has a supply of vegetables that cannot be exceeded, and each vendor has a demand for vegetables that must be satisfied. A multitude of such problems used in this project are solved using LINGO as follows.

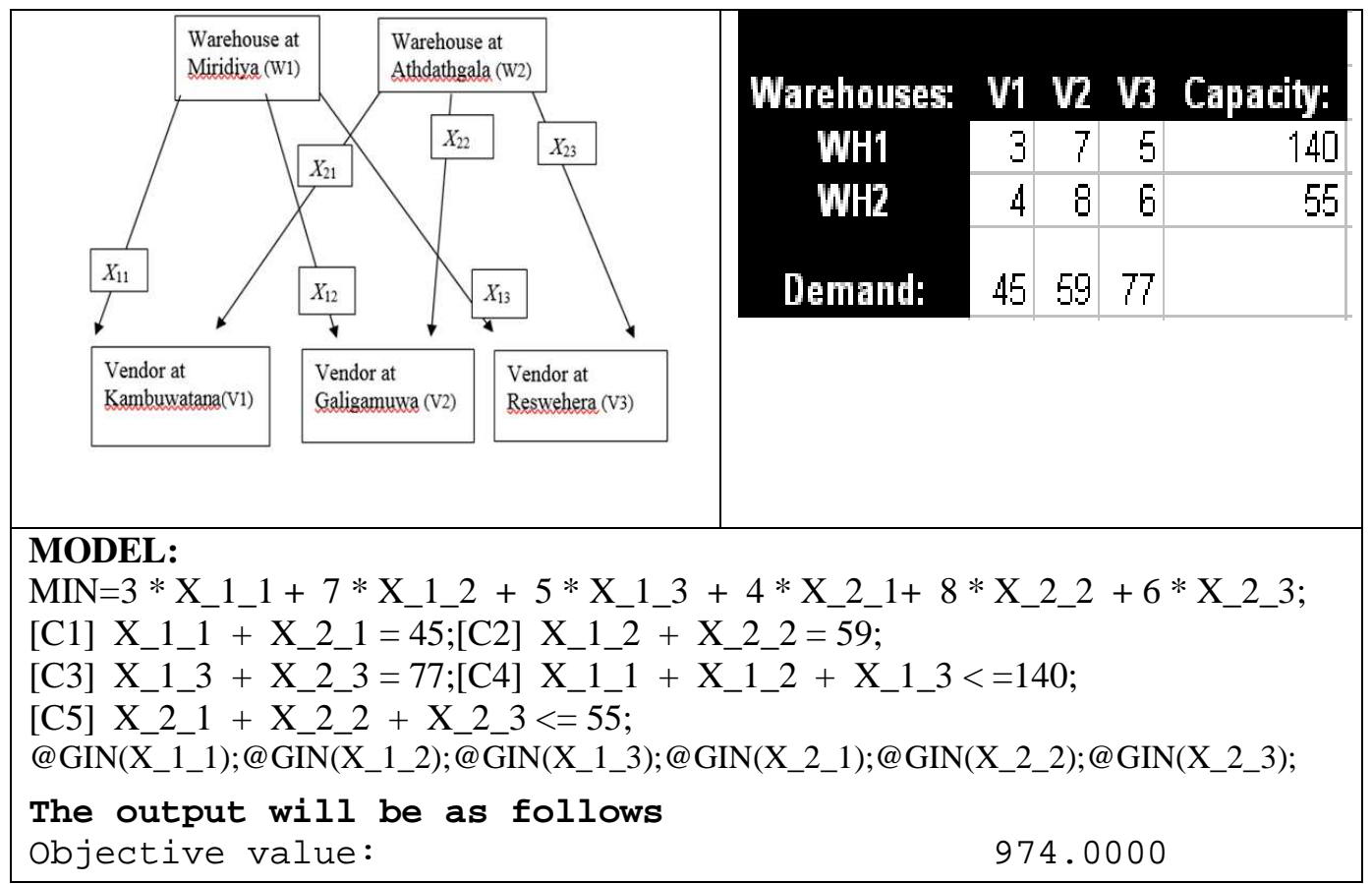

Table 3: Implementation of warehouse/vendor distribution algorithm 


\section{Application of Clusters in a Transportation Network}

\section{Getting started}

A main issue of this research is to find a proper system to transport all vegetables from warehouses to the vendors under optimum conditions. According to the model, one person in each district was assigned to take all demand values of the corresponding district and update the system at a certain time each day, using a secure login protocol. Thus, according to the system, each responsible person of each district should update his data at a given time each day and the route analysis before the route analysis is performed.

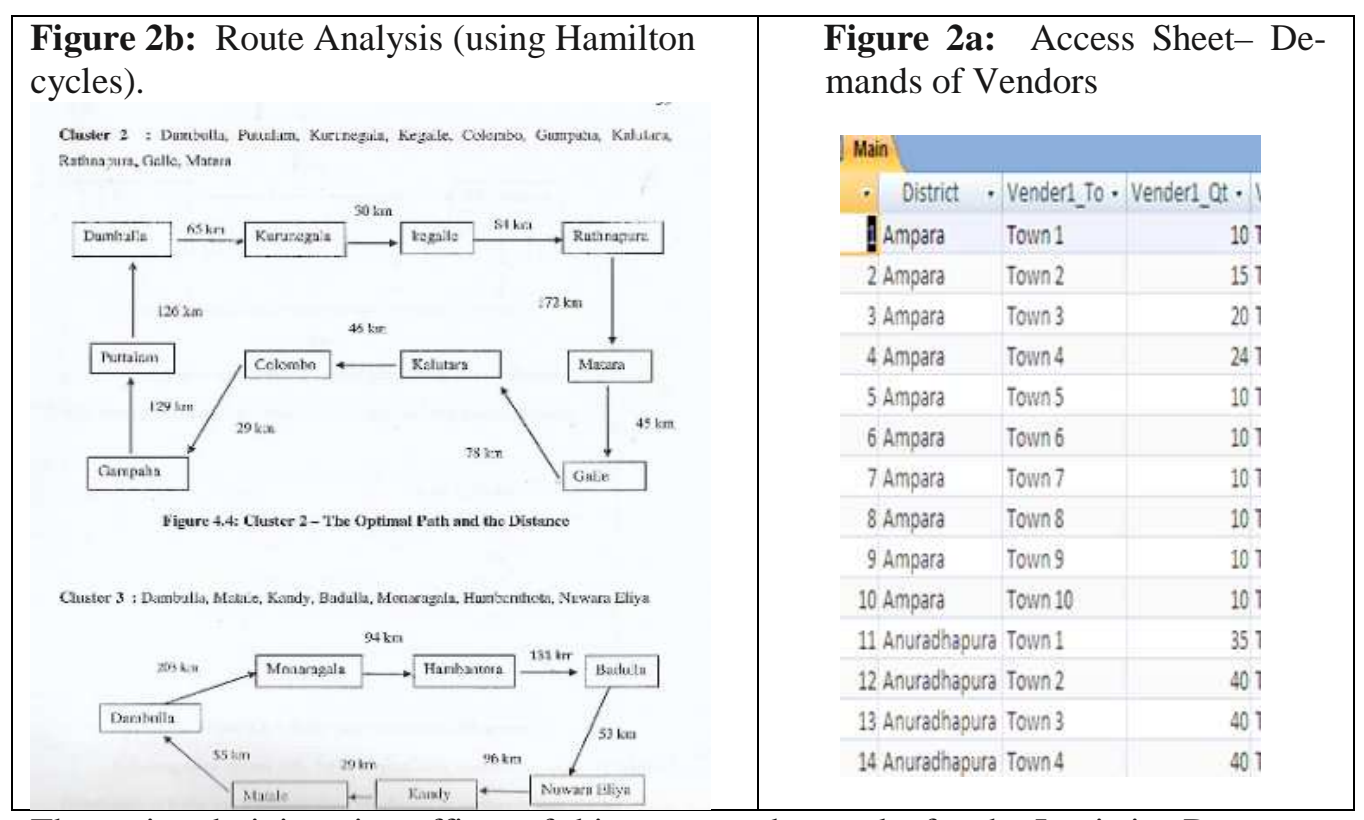

The main administrative officer of this system who works for the Logistics Department

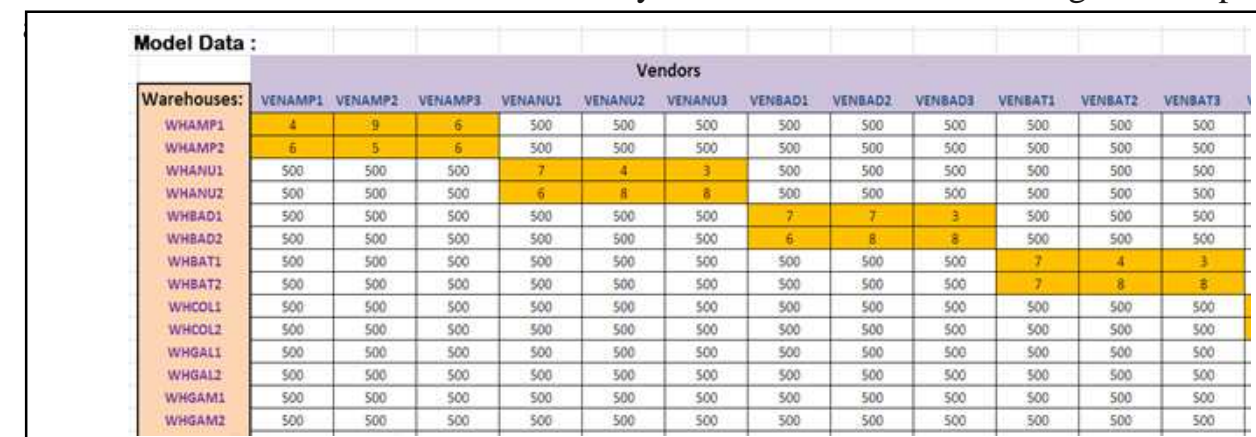

Figure 3: Transportation cost matrix of warehouses and vendors

Shipments

\begin{tabular}{|l|r|r|r|r|r|r|}
\hline Warehouses: VENAMP1 & VENAMP2 & VENAMP3 & VENANU1 & VENANU2 & VENANU3 \\
\hline & 25 & 0 & 0 & 0 & 0 & 0 \\
\hline & 0 & 0 & 0 & 0 & 262 & 0 \\
\hline & 0 & 0 & 0 & 0 & 0 & 120 \\
\hline & 0 & 0 & 0 & 120 & 0 & 0 \\
\hline
\end{tabular}

Figure 4: Solution set within a cluster and the Lingo output 


\section{D.G.N.D.Jayarathna and C.J.Jayawardene}

The LINGO software connected to the Excel sheet imports the entered data and analyzes them using a LINGO package. The analyzed data is then distributed to the respective users via a Java ODBC bridge (as an Access Database). The Java code for Database Connectivity to access using an Action Performed function is run by the main administrative officer at Dambulla.

This enables the responsible person of each district to have access to this system and find the optimal schedule for transport planning, along with quantities of shipment within designated clusters (see Figure 3 and Figure 4).

\section{Conclusion and recommendation}

This model is flexible enough to be re-scheduled/extended at any request. It helps to create a larger model for solving any type of transportation planning problem.

As the first task, we arranged the optimal path to distribute the vegetables and fruits to the minor hubs. This reduces fuel consumption and unnecessary waste of time. In addition, due to problems that may arise delays inside a given city/drop off point,it is best to update both Hamilton path algorithm and Warehouse Vendor algorithm using the spiting of nodes corresponding to such locations (see figure 4).

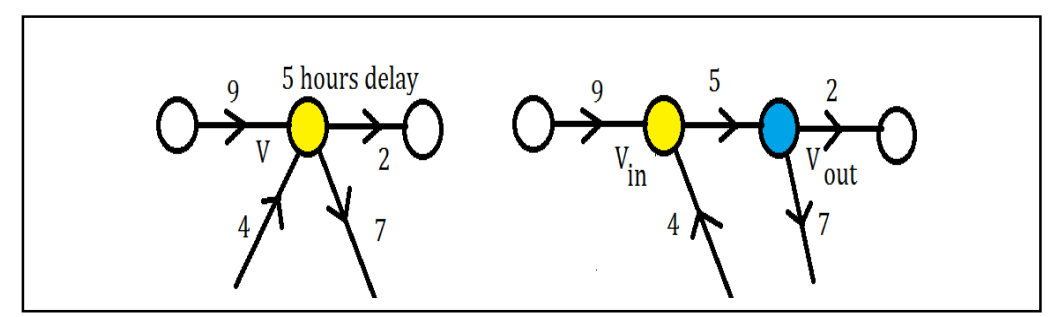

Figure 4: Splitting the node $V$ into $\nu_{\text {in }}$ and $\nu_{\text {out }}$ appropriately.

We recommend carrying out a pilot survey and gravity model to find the optimal locations where new warehouses can be built in each district.

It is recommended to assign one person from each district to submit all sub vendors' requirements. By arranging one person to manage more than one district,it is possible to reduce the cost incurred byMDL in Dambulla.

\section{REFERENCES}

1. M.Bazaraa, J.Jarvis, and H.Sherali, Linear programming and network flow, $2^{\text {nd }}$ edition, Wiley, New York(1990).

2. A.Chanrnes, F.Glover and D.Klingman, A note on a distribution problem, Operations Research, 18(1970) 1213-1216.

3. A.Hakim and R.Kabir, An efficient approach for finding an initial basicf solution for transportation problems, Progress in Nonlinear Dynamics and Chaos, 5(1) (2017) $17-23$.

4. K.Murty, Network programming, Prentice Hall, Upper Saddle River, N.J. (1992),

5. P.Pandian and G.Natarajan, A new approach for solving transportation problems with mixed constraints, Journal of Physical Sciences,14 (2010) 53-61. 
Application of Clusters in a Transportation Network

6. J.Rodrigue and T.Notteboom, Comparative North American and European Gateway Logistics: A regionalism of freight distribution, Journal of Transport Geography, 18(4)(2010) 497-507.

7. C.Yang, L.Zhao, K.Shu and L.Wang, Optimization of bi-clustering algorithm based on greedy randomized adaptive search procedure, Journal of Mathematics and Informatics, 12 (2018) 63-77.

8. X. Yu, A stock model for uncertain European finance markets, Journal of Mathematics and Informatics, 1 (2013-14) 13-24. 